Article

\title{
Nutrition Mediates the Relationship between Osteosarcopenia and Frailty: A Pathway Analysis
}

\author{
Justin Chew ${ }^{1,2, *} \mathbb{C}$, Audrey Yeo ${ }^{2}$, Suzanne Yew ${ }^{2}$, Cai Ning Tan ${ }^{2}$, Jun Pei Lim ${ }^{1,2}$, \\ Noor Hafizah Ismail ${ }^{1,3}$ and Wee Shiong Lim ${ }^{1,2}$ (D) \\ 1 Department of Geriatric Medicine, Tan Tock Seng Hospital, Singapore 308433, Singapore; \\ Jun_Pei_LIM@ttsh.com.sg (J.P.L.); noor_hafizah@ttsh.com.sg (N.H.I.); wee_shiong_lim@ttsh.com.sg (W.S.L.) \\ 2 Institute of Geriatrics and Active Ageing, Tan Tock Seng Hospital, Singapore 308433, Singapore; \\ audrey_jp_yeo@ttsh.com.sg (A.Y.); Suzanne_PY_YEW@ttsh.com.sg (S.Y.); \\ Cai_Ning_TAN@ttsh.com.sg (C.N.T.) \\ 3 Department of Continuing and Community Care, Tan Tock Seng Hospital, Singapore 308433, Singapore \\ * Correspondence: justin_chew@ttsh.com.sg
}

Received: 29 August 2020; Accepted: 23 September 2020; Published: 27 September 2020

check for updates

\begin{abstract}
Osteosarcopenia is associated with increased risk of adverse outcomes such as falls and fractures. Its association with frailty is less well-described, particularly in independent community-dwelling older adults. Although nutrition plays a crucial role in maintaining bone and muscle health, the complex relationship between osteosarcopenia and nutrition in the pathogenesis of frailty remains to be elucidated. In this cross-sectional analysis of 230 independent, community-dwelling individuals (mean age 67.2 \pm 7.4 years), we examined the associations between osteosarcopenia with nutritional status and frailty, and the mediating role of nutrition in the association between osteosarcopenia and frailty. Osteosarcopenia was defined as fulfilling both the Asian Working Group for Sarcopenia 2019 consensus definition (low relative appendicular skeletal muscle mass adjusted for height, in the presence of either of either low handgrip strength or slow gait speed) and T-score $\leq-2.5 \mathrm{SD}$ on bone mineral densitometry. We assessed frailty using the modified Fried criteria and nutrition using the Mini-Nutritional Assessment. We performed multiple linear regression, followed by pathway analysis to ascertain whether nutrition mediates the relationship between osteosarcopenia and frailty. Our study population comprised: 27 (11.7\%) osteosarcopenic, 35 (15.2\%) sarcopenic, 36 (15.7\%) osteoporotic and 132 (57.4\%) normal (neither osteosarcopenic, sarcopenic nor osteoporotic). Osteosarcopenia $(\beta=1.1,95 \% \mathrm{CI} 0.86-1.4)$ and sarcopenia $(\beta=1.1,95 \% \mathrm{CI}$ 0.90-1.4) were significantly associated with frailty, but not osteoporosis. Nutrition mediated the association between osteosarcopenia and frailty (indirect effect estimate 0.09, bootstrap 95\% CI 0.01-0.22). In conclusion, osteosarcopenia is associated with frailty and poorer nutritional status, with nutrition mediating the association between osteosarcopenia and frailty. Our findings support early nutritional assessment and intervention in osteosarcopenia to mitigate the risk of frailty.
\end{abstract}

Keywords: nutrition; osteosarcopenia; frailty

\section{Introduction}

Osteoporosis and sarcopenia are two important musculoskeletal conditions that adversely impact the health of older individuals. While both conditions appear to be distinct, the coexistence of the two conditions, known as osteosarcopenia, may identify a subpopulation of older individuals at greater risk of adverse health outcomes compared to either condition alone. Osteosarcopenia is associated with a 3- to 4-fold increased odds of falls and fractures in older individuals attending a falls clinic [1]. 
Osteosarcopenic individuals who suffered a hip fracture also had 1.8 times greater hazard for mortality compared to non-osteosarcopenic individuals [2].

Despite some studies demonstrating the deleterious effects of osteosarcopenia, others show inconsistent outcomes. For example, community-dwelling older Australian men do not have increased falls, fractures or mortality risk above and beyond either condition alone $[3,4]$. These discrepant findings may be ascribed to differences in the population under study and the chosen outcomes. In a cohort of independent community-dwelling older individuals, frailty is a particularly salient outcome, representing increased vulnerability to adverse events. However, few studies have examined the association between osteosarcopenia and frailty [5-7], relative to osteoporosis and sarcopenia alone.

Nutrition plays a key role in the pathogenesis of frailty, and it is also important in both bone [8] and muscle health [9]. However, the complex relationships between osteosarcopenia, nutrition and frailty remain to be elucidated. Specifically, the mediating role of nutrition in the relationship between osteosarcopenia and frailty bears further scrutiny. Clarifying this relationship will explicate the role of nutrition as a mechanism through which osteosarcopenia engenders frailty, and establish nutrition as an appropriate target for intervention in preventing the adverse consequences of osteosarcopenia.

Therefore, we aimed to, firstly, examine the association between osteosarcopenia, osteoporosis and sarcopenia with frailty and markers of nutritional status. Secondly, we investigated nutrition as a mediator of the relationship between osteosarcopenia and frailty, relative to osteoporosis and sarcopenia alone, in a cohort of independent community-dwelling older adults. We hypothesize that osteosarcopenia is associated with frailty and markers of poor nutritional status, and that the relationship between osteosarcopenia and frailty is mediated by nutrition.

\section{Materials and Methods}

\subsection{Participants and Setting}

This is a cross-sectional analysis of 230 participants recruited from the "Longitudinal Assessment of Biomarkers for characterization of early Sarcopenia and Osteosarcopenic Obesity in predicting frailty and functional decline in community-dwelling Asian older adults Study" (GeriLABS 2), a prospective cohort study involving community-dwelling cognitively intact and functionally independent adults aged 50 years and older [10]. Inclusion criteria were: (i) participants aged 50 to 99 years at study enrollment, (ii) community-dwelling, (iii) independent in both activities of daily living (ADLs) and instrumental ADLs, and (iv) non-frail as defined by the FRAIL criteria [11]. We excluded participants with dementia or evidence of cognitive impairment (modified Chinese version of Mini-Mental State Examination (CMMSE) score $\leq 21$ ) [12]; who were unable to walk 8-m independently; or living in a sheltered or nursing home. All participants provided written informed consent to participate in the study. Ethics approval for this study was obtained from the Domain Specific Review Board of the National Healthcare Group (DSRB Ref: 2017/00850).

\subsection{Data Collection}

We collected demographic characteristics (age, gender, ethnicity) and comorbid conditions. Cognitive performance was assessed using the modified Chinese version of Mini-Mental State Examination (CMMSE). Functional status was evaluated using Barthel's basic activities of daily living (BADL) index [13] and Lawton and Brody's instrumental ADL (IADL) index [14]. Depressive symptoms were evaluated using the Geriatric Depression Scale (GDS) [15]. 
Body composition and bone mineral density (BMD) of lumbar spine, femoral neck and total hip of all participants were obtained with the same dual energy X-ray absorptiometry (DXA) source (Discovery ${ }^{\mathrm{TM}}$ APEX 13.3; Hologic, Bedford, MA, USA). Osteoporosis was classified by the WHO (World Health Organization) criteria based on the BMD T-score of the lumbar spine and/or femoral neck and/or total hip equivalent or lower than -2.5 SD [16]. Sarcopenia was diagnosed with the Asian Working Group for Sarcopenia (AWGS) 2019 consensus criteria [17], defined as low relative appendicular skeletal muscle mass (ASM) (height-adjusted ASM (ASM/height $\left.{ }^{2}\right)$ ) in the presence of either low handgrip strength or slow gait speed using updated cutoffs [18]. Osteosarcopenia was defined as co-existent osteoporosis and sarcopenia.

Physical frailty was assessed using the modified Fried phenotypic criteria [19]. The modified Fried criteria was operationalized as follows [20]: (1) Body mass index less than 18.5; (2) handgrip strength range $<28 \mathrm{~kg}$ for men and $<18 \mathrm{~kg}$ for women, measured using a hydraulic hand dynamometer (North Coast Exacta ${ }^{\mathrm{TM}}$ Hydraulic Hand Dynamometer; North Coast Medical, Inc., Morgan Hill, CA, USA); (3) usual gait speed $<1.0 \mathrm{~m} / \mathrm{s}$ on the $3 \mathrm{~m}$ walk test; (4) low physical activity defined using the pentile cutoff of $\leq 29$ on the Frenchay Activities Index [21]; and (5) fatigue endorsed on either of two questions from the Center for Epidemiologic Studies-Depression Scale (CES-D) modified to assess fatigue. The five items were added to yield a total score for frailty (range $0-5$ ). Higher scores indicated worsening frailty status.

Nutritional assessments included anthropometric measurements: standing height and body weight were measured to calculate body mass index (BMI), waist circumference (WC), mid-arm circumference (MAC), and calf circumference (CC). WC, MAC and CC were measured using a non-elastic tape. WC was measured at $2.5 \mathrm{~cm}$ above the umbilicus. MAC of the dominant arm was obtained with the subject seated, measured at the midpoint between the tip of the shoulder and the tip of the elbow. CC was obtained with the subject in a seated position with both feet on the ground to obtain the maximal reading of the dominant calf [10]. To evaluate nutritional status, the Mini Nutritional Assessment (MNA) [22] was also used. In accordance with established cut-offs, a total MNA score of $\geq 24$ (out of a maximum of 30 points) classifies well-nourished/normal nutritional status, 17 to 23.5 points classifies individuals at risk of malnutrition, and $<17$ points malnourished. Lastly, serum 25-hydroxyvitamin D (25(OH)D) levels were also obtained.

\subsection{Statistical Analysis}

Continuous variables were expressed as means and standard deviation. Categorical variables were expressed as counts and percentages. One-way analysis of variance (ANOVA) followed by Bonferroni post hoc analyses were used to analyse the relationships of continuous variables (Frailty score and nutritional parameters) with participants classified as osteoporotic, sarcopenic, osteosarcopenic or normal (neither osteoporotic, sarcopenic nor osteosarcopenic) respectively. Multiple linear regression was carried out with frailty score and the aforementioned nutritional parameters as dependent variables, unadjusted and adjusted for age, gender and ethnicity.

A multicategorical mediation model examined the potential mediating role of nutrition, using total MNA score, in the relationship between osteoporosis, sarcopenia and osteosarcopenia with frailty. Dummy codes were created for osteoporosis, sarcopenia and osteosarcopenia with normal individuals (neither osteoporotic, sarcopenic nor osteosarcopenic) as the reference group (Figure 1). Direct and indirect effects for osteoporosis, sarcopenia and osteosarcopenia groups were compared to this reference group. To obtain estimates of the indirect effects, we used bootstrapping procedures based on 5000 resamples [23]. Participants' age, gender and ethnicity were included in the model as covariates. Two-sided tests with a significance level of $p<0.05$ were applied. Statistical analysis was carried out using Stata version 13 (StataCorp, College Station, TX, USA). 


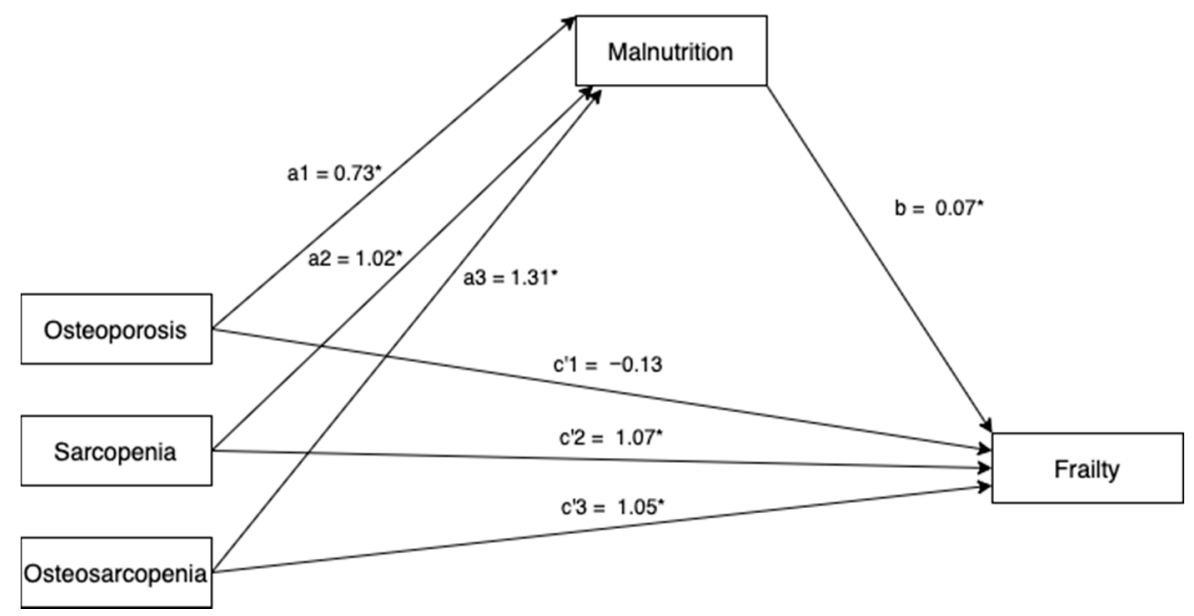

Figure 1. Multicategorical mediation model for total frailty score $\left({ }^{*} p<0.05\right)$. Paths a1, a2, a3 represent the effect of osteoporosis, sarcopenia and osteosarcopenia on the proposed mediator, malnutrition; path $b$ represents the effect of the proposed mediator, malnutrition on frailty; path $c^{\prime} 1, c^{\prime} 2$ and $c^{\prime} 3$ represent the relative direct effect of osteoporosis, sarcopenia and osteosarcopenia on frailty. All paths are represented by unstandardized regression coefficients.

\section{Results}

\subsection{Baseline Characteristics}

We included 230 individuals (mean age $67.2 \pm 7.4$ years) in this study. Twenty-seven $(11.7 \%)$ were osteosarcopenic, 35 (15.2\%) were sarcopenic, 36 (15.7\%) were osteoporotic and 132 (57.4\%) were normal (neither osteosarcopenic, sarcopenic nor osteoporotic). A significant difference in age was observed between the four groups $(p=0.0005)$; post-hoc comparisons with Bonferroni correction revealed that osteosarcopenic $(71.4 \pm 6.9$ years, $p=0.001)$ and sarcopenic individuals $(69.3 \pm 7.1$ years, $p=0.048)$ were significantly older compared to normal individuals ( $65.7 \pm 7.3$ years). There were no significant differences observed in gender, comorbidities, ADL function, cognitive function, depressive symptoms and Vitamin D levels between the groups (Table 1).

Table 1. Baseline characteristics, nutritional and frailty status.

\begin{tabular}{|c|c|c|c|c|c|}
\hline Characteristics & Normal $(N=132)$ & $\mathrm{O}(N=36)$ & $\mathrm{S}(N=35)$ & OS $(N=27)$ & $p$ \\
\hline Age in years, mean (SD) & $65.7(7.3)$ & $67.6(7.0)$ & $69.3(7.1) *$ & $71.4(6.9)^{*}$ & 0.0005 \\
\hline Female gender, N (\%) & $99(75)$ & $30(83.3)$ & $23(65.7)$ & $15(55.6)$ & 0.064 \\
\hline Chinese ethnicity, N (\%) & $116(87.9)$ & $36(100)$ & $35(100)$ & $25(92.6)$ & 0.024 \\
\hline \multicolumn{6}{|l|}{ Comorbidities, N (\%) } \\
\hline Hypertension & $44(33.3)$ & $13(36.1)$ & $15(42.9)$ & $10(37.0)$ & 0.77 \\
\hline Hyperlipidemia & $69(52.3)$ & $23(63.9)$ & $19(54.3)$ & $19(70.4)$ & 0.27 \\
\hline Diabetes mellitus & $13(9.9)$ & $7(19.4)$ & $8(22.9)$ & $5(15.8)$ & 0.15 \\
\hline Ischemic heart disease & $3(2.3)$ & $1(2.8)$ & $1(2.9)$ & 0 & 0.86 \\
\hline Stroke or transient ischemic attack & $3(2.3)$ & 0 & $1(2.9)$ & 0 & 0.66 \\
\hline Kidney disease & $7(5.3)$ & 0 & $2(5.7)$ & 0 & 0.31 \\
\hline Asthma & $10(7.6)$ & $1(2.8)$ & $3(8.6)$ & $1(3.7)$ & 0.65 \\
\hline Arthritis & $16(12.1)$ & $1(2.8)$ & $5(14.3)$ & $3(11.1)$ & 0.38 \\
\hline \multicolumn{6}{|l|}{ Physical function, mean (SD) } \\
\hline ADL & $98.0(3.3)$ & $98.9(3.0)$ & $98.3(3.0)$ & $97.4(4.2)$ & 0.31 \\
\hline \multicolumn{6}{|l|}{ Cognitive function, mean (SD) } \\
\hline CMMSE & $26.3(1.7)$ & $25.9(1.8)$ & $25.7(1.8)$ & $26.2(1.8)$ & 0.30 \\
\hline \multicolumn{6}{|l|}{ Depressive symptoms, mean (SD) } \\
\hline GDS & $1.1(1.8)$ & $1.1(2.0)$ & $1.2(1.1)$ & $1.2(0.9)$ & 0.99 \\
\hline \multicolumn{6}{|l|}{ Nutrition and anthropometry, mean (SD) } \\
\hline MNA total score & $27.6(1.7)$ & $26.8(1.9)$ & $26.7(2.0)$ & $26.5(1.9) *$ & 0.0035 \\
\hline
\end{tabular}


Table 1. Cont.

\begin{tabular}{llllll}
\hline Characteristics & Normal $(N=132)$ & $\mathbf{O}(N=36)$ & $\mathbf{S}(N=35)$ & OS $(N=27)$ & $p$ \\
\hline MNA categories, $\mathrm{N}(\%)$ & & & & & 0.22 \\
$\quad$ At risk & $4(3.0)$ & $4(11.1)$ & $3(8.6)$ & $2(7.4)$ & \\
$\quad$ Normal & $128(97.0)$ & $32(88.9)$ & $32(91.4)$ & $25(92.6)$ & \\
\hline BMI $\left(\mathrm{kg} / \mathrm{m}^{2}\right)$ & $24.8(3.4)$ & $22.9(2.8)^{*}$ & $22.5(2.2)^{*}$ & $22.3(2.5)^{*}$ & $<0.001$ \\
\hline Calf circumference $(\mathrm{cm})$ & $35.8(3.0)$ & $34.5(2.7)$ & $33.3(2.5)^{*}$ & $32.3(3.6)^{* * *}$ & $<0.001$ \\
\hline Mid-arm circumference $(\mathrm{cm})$ & $28.4(2.8)$ & $26.9(2.9)$ & $27.0(3.0)^{*}$ & $25.6(2.6)^{* * * *}$ & $<0.001$ \\
\hline Waist circumference $(\mathrm{cm})$ & $86.9(10.0)$ & $83.2(8.9)$ & $84.2(7.0)$ & $81.7(7.7)^{*}$ & 0.015 \\
\hline DEXA fat $\%$ & $38.8(6.2)$ & $38.2(6.0)$ & $37.5(7.2)$ & $36.1(7.6)$ & 0.22 \\
\hline Serum Vitamin D levels $(\mu \mathrm{g} / \mathrm{L})$ & $29.9(8.5)$ & $30.4(80)$ & $31.9(10.2)$ & $32.7(9.3)$ & 0.38 \\
\hline Fried Frailty total score, mean $(\mathrm{SD})$ & $0.38(0.64)$ & $0.33(0.68)$ & $1.6(0.81)^{* / * *}$ & $1.7(0.72)^{* * * *}$ & $<0.001$ \\
\hline O osteopor & & &
\end{tabular}

O, osteoporosis; S, sarcopenia; OS, osteosarcopenia; SD, standard deviation; ADL, activities of daily living; CMMSE, modified version of the Chinese Mini-Mental State Examination; GDS, Geriatric Depression Scale; MNA, Mini-Nutritional Assessment; BMI, body mass index; DEXA, dual-energy x-ray absorptiometry. ${ }^{*} p<0.05$ vs. normal; ${ }^{* *} p<0.05$ vs. osteoporosis.

\subsection{Association between Osteoporosis, Sarcopenia and Osteosarcopenia with Frailty}

There was a statistically significant difference in frailty score between the four groups $(p<0.001)$. Post hoc analyses with Bonferonni correction revealed that frailty score was significantly higher in participants with osteosarcopenia $(1.7 \pm 0.72, p<0.001)$ and sarcopenia $(1.6 \pm 0.81, p<0.001)$ compared to those who were normal $(0.38 \pm 0.64)$. There were no differences in frailty score between osteoporotic-only and normal individuals. There were also no differences in frailty score between osteosarcopenic and sarcopenic individuals (Table 1).

In a multiple linear regression model adjusted for age, gender and ethnicity, sarcopenia $(\beta=1.15$, 95\% CI 0.90-1.4, $p<0.001)$ and osteosarcopenia $(\beta=1.14,95 \%$ CI $0.86-1.4, p<0.001)$ were associated with frailty, but not osteoporosis ( $\beta=-0.077,95 \%$ CI $-0.32-0.17, p=0.54$ ) (Table 2).

Table 2. Multiple linear regression models with total frailty score as the dependent variable.

\begin{tabular}{lllllllll}
\hline & \multicolumn{7}{c}{ Unadjusted Model } & \multicolumn{7}{c}{ Adjusted Model * } \\
\hline Total Frailty Score & $\boldsymbol{\beta}$ & SE & $\mathbf{9 5 \%}$ CI & $\boldsymbol{p}$ & $\boldsymbol{\beta}$ & $\mathbf{S E}$ & $\mathbf{9 5 \%}$ CI & $\boldsymbol{p}$ \\
\hline Osteoporosis & -0.045 & 0.13 & $-0.30-0.21$ & 0.72 & -0.077 & 0.12 & $-0.32-0.17$ & 0.54 \\
Sarcopenia & 1.25 & 0.13 & $0.99-1.50$ & $<0.001$ & 1.15 & 0.13 & $0.90-1.40$ & $<0.001$ \\
Osteosarcopenia & 1.32 & 0.14 & $1.04-1.61$ & $<0.001$ & 1.14 & 0.14 & $0.86-1.42$ & $<0.001$ \\
\hline & $R^{2}=0.057$ & \multicolumn{7}{c}{$R^{2}=0.50$} \\
\hline
\end{tabular}

$\mathrm{SE}$, standard error; CI, confidence interval. * Adjusted for age, gender and ethnicity.

\subsection{Association between Osteoporosis, Sarcopenia and Osteosarcopenia with Nutrition}

There were statistically significant differences in total MNA score, BMI, WC, MAC and CC between the four groups. MNA total score was significantly lower in osteosarcopenic $(26.5 \pm 1.9)$ compared to normal individuals $(27.6 \pm 1.7, p=0.028)$. In the whole cohort, using established cutoffs for MNA total score, there were no individuals classified as malnourished, $13(5.7 \%)$ were classified as "at risk" of malnutrition, and 217 (94.4\%) were classified as normal. There were no significant differences in MNA categories between the four groups. BMI was significantly lower in osteosarcopenic $\left(22.3 \pm 2.5 \mathrm{~kg} / \mathrm{m}^{2}\right.$, $p=0.001)$, sarcopenic $\left(22.5 \pm 2.2 \mathrm{~kg} / \mathrm{m}^{2}, p=0.001\right)$ and osteoporotic individuals $\left(22.9 \pm 2.8 \mathrm{~kg} / \mathrm{m}^{2}\right.$, $p=0.005)$ compared to normal individuals $\left(24.8 \pm 3.4 \mathrm{~kg} / \mathrm{m}^{2}\right)$. WC and MAC were also lower in osteosarcopenic compared to normal individuals (WC: $81.7 \pm 7.7 \mathrm{~cm}$ versus $86.9 \pm 10.0 \mathrm{~cm}, p=0.045$; MAC: $25.6 \pm 2.6 \mathrm{~cm}$ versus $28.4 \pm 2.8 \mathrm{~cm}, p<0.001$ ). CC was also significantly lower in osteosarcopenic $(32.3 \pm 3.6 \mathrm{~cm}, p<0.001)$ and sarcopenic individuals $(33.3 \pm 2.5 \mathrm{~cm}, p<0.001)$ compared to normal individuals $(35.8 \pm 3.0 \mathrm{~cm})$ (Table 1$)$.

Osteosarcopenia was significantly associated with total MNA score, BMI, CC, WC and MAC in multiple linear regression models, unadjusted and adjusted for age, gender and ethnicity. 
The coefficients observed were of a greater magnitude for individuals with osteosarcopenia, compared to individuals with osteoporosis or sarcopenia alone (Table 3).

Table 3. Multiple linear regression models with nutritional parameters as dependent variables.

\begin{tabular}{|c|c|c|c|c|c|c|c|c|c|c|c|c|c|c|c|}
\hline \multirow[t]{2}{*}{$\begin{array}{l}\text { Unadjusted } \\
\text { Models }\end{array}$} & \multicolumn{3}{|c|}{ MNA (Total Score) } & \multicolumn{3}{|c|}{ BMI, kg/m² } & \multicolumn{3}{|c|}{$\begin{array}{l}\text { Calf } \\
\text { Circumference, cm }\end{array}$} & \multicolumn{3}{|c|}{$\begin{array}{l}\text { Waist } \\
\text { Circumference, cm }\end{array}$} & \multicolumn{3}{|c|}{$\begin{array}{l}\text { Mid-Arm } \\
\text { Circumference, cm }\end{array}$} \\
\hline & $\beta$ & SE & $p$ & $\beta$ & SE & $p$ & $\beta$ & SE & $p$ & $\beta$ & SE & $p$ & $\beta$ & SE & $p$ \\
\hline Osteoporosis & -0.73 & 0.34 & 0.035 & -1.95 & 0.57 & 0.001 & -1.25 & 0.56 & 0.026 & -3.71 & 1.72 & 0.032 & -1.52 & 0.54 & 0.005 \\
\hline Sarcopenia & -0.89 & 0.35 & 0.011 & -2.3 & 0.58 & $<0.001$ & -2.43 & 0.56 & $<0.001$ & -2.75 & 1.74 & 0.116 & -1.4 & 0.54 & 0.011 \\
\hline \multirow[t]{2}{*}{ Osteosarcopenia } & -1.1 & 0.39 & 0.005 & -2.57 & 0.64 & $<0.001$ & 3.49 & 0.62 & $<0.001$ & -5.22 & 1.94 & 0.008 & -2.84 & 0.6 & $<0.001$ \\
\hline & \multicolumn{3}{|c|}{$\mathrm{R}^{2}=0.058$} & \multicolumn{3}{|c|}{$\mathrm{R}^{2}=0.12$} & \multicolumn{3}{|c|}{$\mathrm{R}^{2}=0.16$} & \multicolumn{3}{|c|}{$R^{2}=0.045$} & \multicolumn{3}{|c|}{$\mathrm{R}^{2}=0.11$} \\
\hline \multirow[t]{2}{*}{$\begin{array}{l}\text { Adjusted } \\
\text { Models * }\end{array}$} & \multicolumn{3}{|c|}{ MNA (Total Score) } & \multicolumn{3}{|c|}{ BMI, kg/m² } & \multicolumn{3}{|c|}{$\begin{array}{l}\text { Calf } \\
\text { Circumference, } \mathrm{cm}\end{array}$} & \multicolumn{3}{|c|}{$\begin{array}{l}\text { Waist } \\
\text { Circumference, cm }\end{array}$} & \multicolumn{3}{|c|}{$\begin{array}{l}\text { Mid-Arm } \\
\text { Circumference, cm }\end{array}$} \\
\hline & $\beta$ & SE & $p$ & $\beta$ & SE & $p$ & $\beta$ & SE & $p$ & $\beta$ & SE & $p$ & $\beta$ & SE & $p$ \\
\hline Osteoporosis & -0.73 & 0.35 & 0.037 & -1.94 & 0.57 & 0.001 & -1.07 & 0.56 & 0.057 & -3.05 & 1.64 & 0.063 & -1.44 & 0.54 & 0.008 \\
\hline Sarcopenia & -1.02 & 0.36 & 0.005 & -2.39 & 0.59 & $<0.001$ & -2.44 & 0.57 & $<0.001$ & -3.36 & 1.67 & 0.046 & -1.52 & 0.56 & 0.007 \\
\hline \multirow[t]{2}{*}{ Osteosarcopenia } & -1.31 & 0.4 & 0.001 & -2.91 & 0.66 & $<0.001$ & -3.65 & 0.64 & $<0.001$ & -7.24 & 1.87 & $<0.001$ & -3.12 & 0.62 & $<0.001$ \\
\hline & \multicolumn{3}{|c|}{$\mathrm{R}^{2}=0.082$} & \multicolumn{3}{|c|}{$R^{2}=0.16$} & \multicolumn{3}{|c|}{$\mathrm{R}^{2}=0.19$} & \multicolumn{3}{|c|}{$\mathrm{R}^{2}=0.18$} & \multicolumn{3}{|c|}{$\mathrm{R}^{2}=0.13$} \\
\hline
\end{tabular}

$\mathrm{SE}$, standard error; $\mathrm{CI}$, confidence interval. * Adjusted for age, gender and ethnicity.

\subsection{Mediating Effect of Nutrition on the Relationship between Osteoporosis, Sarcopenia and Osteosarcopenia} with Frailty

In the multicategorical mediation model, there were significant relationships between osteoporosis (a1 coefficient $(\mathrm{SE})=-0.73(0.34), p=0.033)$, sarcopenia (a2 coefficient (SE) $=-1.02(0.35), p=0.004)$ and osteosarcopenia (a3 coefficient $(\mathrm{SE})=-1.31(0.39), p=0.001$ ) with total MNA scores. The mediating variable, total MNA score, was significantly related to total frailty score (b coefficient $(\mathrm{SE})=-0.07(0.02)$, $p=0.002$ ). Bootstrapping revealed significant relative indirect effects for osteoporosis (estimate $=0.05$, bootstrap 95\% CI 0.007-0.14), sarcopenia (estimate $=0.07$, bootstrap 95\% CI $0.02-0.17$ ) and osteosarcopenia (estimate $=0.09$, bootstrap 95\% CI 0.01-0.2), indicating that nutrition mediated the association between osteoporosis, sarcopenia and osteosarcopenia, relative to normal individuals. The proportion of the total effect, mediated by nutrition, is $10 \%$ (Table 4 ).

Table 4. Multicategorical mediation model: relative direct, total and indirect effects for the mediating role of nutrition on the relationship between osteosarcopenia, osteoporosis and sarcopenia with frailty.

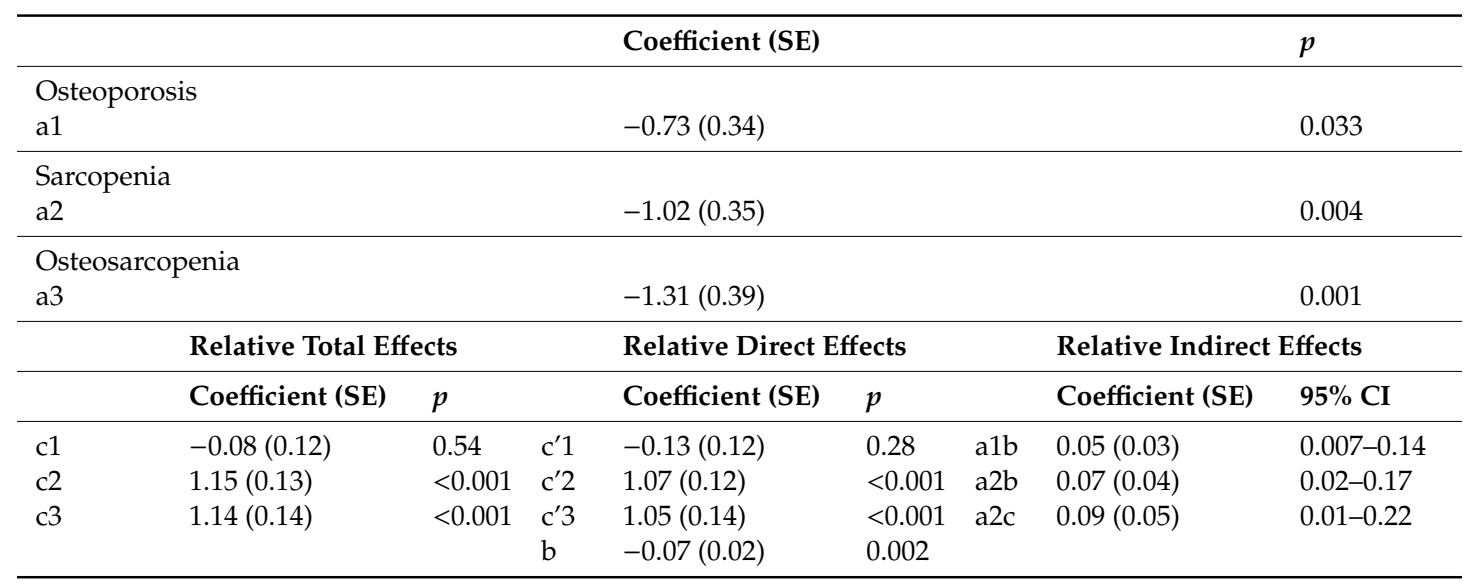




\section{Discussion}

Our study demonstrated that in relatively healthy community-dwelling older adults, osteosarcopenia is present in $11.7 \%$ of individuals and is associated with markers of nutrition, including lower MNA scores, BMI, calf, mid-arm and waist circumference. In the association between osteosarcopenia, sarcopenia and osteoporosis with frailty, we observed a mediating effect of nutrition, which was stronger in osteosarcopenic individuals compared to individuals with sarcopenia or osteoporosis alone.

The prevalence of osteosarcopenia in our study lies within previous estimates in Asian populations, which ranges from $6.2 \%$ [24] to $19.6 \%$ [25]. In contrast, in individuals attending falls clinics or with osteoporotic fractures, prevalence estimates are substantially higher, ranging up to $58 \%$ [26]. These differences in the population studied may account for inconsistent associations between osteosarcopenia and negative outcomes.

In our cohort of relatively younger, independent community-dwelling individuals, we found a significant association between sarcopenia and frailty, with and without concomitant osteoporosis. Both sarcopenia and osteoporosis contribute to frailty through common pathways, including chronic inflammation, which exacerbates muscle [27] and bone loss [28]. Mechanisms include the accumulation and altered distribution of body fat into intra- and intermuscular adipose tissue [29], altering lipid metabolism and promoting the accumulation of macrophages and other immune cells, and increasing the secretion of proinflammatory cytokines [30]. Together, these contribute to a state of chronic, low-grade inflammation favoring the development and progression of sarcopenia. Similarly, in older women, higher levels of inflammatory markers (soluble interleukin-6, tumor necrosis factor receptors 1 and 2) were associated with increased risk of hip fractures, and this association was mediated by BMD [31], further supporting the role of inflammation in both sarcopenia and osteoporosis.

Metabolic and hormonal pathways, particularly in the growth hormone (GH)/insulin-like growth factor-1 (IGF-1) axis, are essential for metabolic regulation of bone and muscle mass, with the decline in GH and IGF-1 levels associated with both osteoporosis and sarcopenia [32]. The dysregulation of this pathway with ageing may also play a role in the pathogenesis of frailty [20], with a study showing an independent association of low levels of IGF-1 with pre-frailty and frailty [33]. Nevertheless, we did not find any associations between osteoporosis alone and frailty. Furthermore, the strength of the association between osteosarcopenia and frailty was also similar to that of sarcopenia and frailty, highlighting the relative importance of interventions to improve muscle health in this cohort to mitigate frailty.

The present study reveals an association between osteosarcopenia, sarcopenia and osteoporosis with markers of nutritional status, with poorer nutritional status observed with osteosarcopenia, compared to isolated sarcopenia and osteoporosis. These findings are consistent with a previous study of geriatric inpatients [34] and individuals attending a falls and fractures clinic [35]. Our finding that nutrition mediates the association between osteosarcopenia and frailty further underscores the crucial role of nutrition in the development of frailty in at-risk individuals with osteosarcopenia. Specifically, loss of muscle mass and increased risk of osteoporosis are associated with caloric deficit, protein and Vitamin D deficiency, which in turn are modifiable nutritional risk factors for frailty [36]. Thus, our findings further provide the impetus for nutritional assessment and early supplementation in osteosarcopenia to ameliorate the detrimental effects of frailty. However, in this cohort, the proportion of the total effect mediated by nutrition is small, suggesting that other mechanisms, such as physical activity or social factors [37], are also important to prevent the development of frailty.

In our cohort of relatively healthy, independent, community-dwelling older adults, there were no individuals classified as malnourished using established cutoffs on the MNA total score. Items in the MNA also overlap with components included in definitions of sarcopenia and frailty, although the effect of the potential "circularity" is likely to be limited, as the $\mathrm{R}^{2}$ values in the unadjusted regression models for BMI, CC and MAC are not large. Despite these observations, our results still demonstrate the associations between osteosarcopenia, malnutrition and frailty, albeit with malnutrition having 
only a small mediating effect on this association. A possible explanation is that the MNA does not provide accurate quantification of protein, caloric and micronutrient intake relevant to bone and muscle health, but instead provides a global assessment of malnutrition risk. This suggests that in independent community-dwelling older adults presenting upstream of the spectrum of frailty, developing nutritional screening tools which are more sensitive to milder degrees of malnutrition (e.g., detection of anorexia as a forward indicator of malnutrition) [38], or with components specific to bone and muscle health may better identify individuals at risk of osteosarcopenia, and subsequent development of frailty.

The strengths of this study include the analysis of a reasonably-sized cohort of independent community-dwelling older adults, the use of contemporary consensus definitions for sarcopenia in an Asian population, and comprehensive evaluation of nutritional status. To our knowledge, this is the first study to use mediation analysis to explicate nutrition as a mechanism underlying the link between osteosarcopenia and frailty. Weaknesses include the cross-sectional nature of this study, which preclude definitive conclusions about causality. The proposed pathway is also exploratory and not exhaustive, and needs to be corroborated in well-designed longitudinal studies. The possibility of residual confounding factors, such as physical activity, which was not measured in this study, also exists. Lastly, we are unable to generalize our results to other older, frailer populations.

In summary, the present study showed that sarcopenia, with and without osteoporosis, is associated with increased frailty, and that nutrition mediates the association between osteosarcopenia and frailty. These findings are salient to older adults living amidst the COVID-19 pandemic, as the need for physical distancing and social isolation may adversely impact nutritional status [39]. Taken together, our results support the need for early identification and interventions for malnutrition in at-risk older adults with osteosarcopenia to prevent the downstream cascade of frailty and disability.

Author Contributions: Data curation, A.Y., S.Y. and C.N.T.; Formal analysis, J.C.; Methodology, J.C. and W.S.L.; Project administration, A.Y., S.Y. and C.N.T.; Supervision, W.S.L.; Writing-original draft, J.C.; Writing一review \& editing, J.C., J.P.L., N.H.I. and W.S.L. All authors have read and agreed to the published version of the manuscript.

Funding: This research was funded by the Lee Foundation.

Acknowledgments: We would like to thank all the participants of this study.

Conflicts of Interest: The authors declare no conflict of interest.

\section{References}

1. Sepúlveda-Loyola, W.; Phu, S.; Hassan, E.B.; Brennan-Olsen, S.L.; Zanker, J.; Vogrin, S.; Conzade, R.; Kirk, B.; Al Saedi, A.; Probst, V.; et al. The Joint Occurrence of Osteoporosis and Sarcopenia (Osteosarcopenia): Definitions and Characteristics. J. Am. Med. Dir. Assoc. 2020, 21, 220-225. [CrossRef]

2. Yoo, J.-I.; Kim, H.; Ha, Y.-C.; Kwon, H.-B.; Koo, K.-H. Osteosarcopenia in Patients with Hip Fracture Is Related with High Mortality. J. Korean Med. Sci. 2018, 33, e27. [CrossRef]

3. Scott, D.; Seibel, M.; Cumming, R.; Naganathan, V.; Blyth, F.; Le Couteur, D.; Handelsman, D.J.; Waite, L.M.; Hirani, V. Does Combined Osteopenia/Osteoporosis and Sarcopenia Confer Greater Risk of Falls and Fracture than Either Condition Alone in Older Men? The Concord Health and Ageing in Men Project. J. Gerontol. Ser. A Biol. Sci. Med. Sci. 2018, 74, 827-834. [CrossRef]

4. Balogun, S.; Winzenberg, T.M.; Wills, K.E.; Scott, D.; Callisaya, M.L.; Cicuttini, F.; Jones, G.; Aitken, D. Prospective associations of osteosarcopenia and osteodynapenia with incident fracture and mortality over 10 years in community-dwelling older adults. Arch. Gerontol. Geriatr. 2019, 82, 67-73. [CrossRef]

5. Yoshimura, N.; Muraki, S.; Oka, H.; Iidaka, T.; Kodama, R.; Horii, C.; Kawaguchi, H.; Nakamura, K.; Akune, T.; Tanaka, S. Do sarcopenia and/or osteoporosis increase the risk of frailty? A 4-year observation of the second and third ROAD study surveys. Osteoporos. Int. 2018, 29, 2181-2190. [CrossRef]

6. Frisoli, A., Jr.; Chaves, P.H.; Ingham, S.J.M.; Fried, L.P. Severe osteopenia and osteoporosis, sarcopenia, and frailty status in community-dwelling older women: Results from the Women's Health and Aging Study (WHAS) II. Bone 2011, 48, 952-957. [CrossRef] 
7. Greco, E.A.; Pietschmann, P.; Savastano, S. Osteoporosis and Sarcopenia Increase Frailty Syndrome in the Elderly. Front. Endocrinol. 2019, 10, 255. [CrossRef]

8. Siddique, N.; O'Donoghue, M.; Casey, M.C.; Walsh, J. Malnutrition in the elderly and its effects on bone health-A review. Clin. Nutr. ESPEN 2017, 21, 31-39. [CrossRef]

9. De Rui, M.; Inelmen, E.M.; Pigozzo, S.; Trevisan, C.; Manzato, E.; Sergi, G. Dietary strategies for mitigating osteosarcopenia in older adults: A narrative review. Aging Clin. Exp. Res. 2019, 31, 897-903. [CrossRef]

10. Lim, W.S.; Lim, J.P.; Chew, J.; Tan, A.W.K. Influence of Obesity on Diagnostic Accuracy and Optimal Cutoffs for Sarcopenia Screening in Non-Frail Older Adults: A Comparison of SARC-F versus SARC-CalF. J. Nutr. Health Aging 2020, 1-3. [CrossRef]

11. Morley, J.E.; Malmstrom, T.K.; Miller, D.K. A simple frailty questionnaire (FRAIL) predicts outcomes in middle aged African Americans. J. Nutr. Health Aging 2012, 16, 601-608. [CrossRef]

12. Sahadevan, S.; Lim, P.P.J.; Tan, N.J.L.; Chan, S.P. Diagnostic performance of two mental status tests in the older Chinese: Influence of education and age on cut-off values. Int. J. Geriatr. Psychiatry 2000, 15, 234-241. [CrossRef]

13. Mahoney, F.I.; Barthel, D.W. Functional Evaluation: The Barthel Index. MD State Med. J. 1965, 14, 61-65.

14. Lawton, M.P.; Brody, E.M. Assessment of older people: Self-maintaining and instrumental activities of daily living. Gerontologist 1969, 9, 179-186. [CrossRef]

15. Yesavage, J.A.; Brink, T.; Rose, T.L.; Lum, O.; Huang, V.; Adey, M.; Leirer, V.O. Development and validation of a geriatric depression screening scale: A preliminary report. J. Psychiatr. Res. 1982, 17, 37-49. [CrossRef]

16. Kanis, J.A. Assessment of fracture risk and its application to screening for postmenopausal osteoporosis: Synopsis of a WHO report. Osteoporos. Int. 1994, 4, 368-381. [CrossRef]

17. Chen, L.-K.; Woo, J.; Assantachai, P.; Auyeung, T.-W.; Chou, M.-Y.; Iijima, K.; Jang, H.C.; Kang, L.; Kim, M.; Kim, S.; et al. Asian Working Group for Sarcopenia: 2019 Consensus Update on Sarcopenia Diagnosis and Treatment. J. Am. Med. Dir. Assoc. 2020, 21, 300-307. [CrossRef]

18. Auyeung, T.-W.; Arai, H.; Chen, L.K.; Woo, J. Letter to the editor: Normative data of handgrip strength in 26344 older adults-A pooled dataset from eight cohorts in Asia. J. Nutr. Health Aging 2020, 24, 125-126. [CrossRef] [PubMed]

19. Fried, L.P.; Tangen, C.M.; Walston, J.; Newman, A.B.; Hirsch, C.; Gottdiener, J.; Seeman, T.; Tracy, R.; Kop, W.J.; Burke, G.; et al. Frailty in older adults: Evidence for a phenotype. J. Gerontol. Ser. A Boil. Sci. Med. Sci. 2001, 56, M146-M157. [CrossRef] [PubMed]

20. Chew, J.; Tay, L.; Lim, J.P.; Leung, B.P.; Yeo, A.; Yew, S.; Ding, Y.Y.; Lim, W.S. Serum Myostatin and IGF-1 as Gender-Specific Biomarkers of Frailty and Low Muscle Mass in Community-Dwelling Older Adults. J. Nutr. Health Aging 2019, 23, 979-986. [CrossRef] [PubMed]

21. Schuling, J.; De Haan, R.; Limburg, M.; Groenier, K.H. The Frenchay Activities Index. Assessment of functional status in stroke patients. Stroke 1993, 24, 1173-1177. [CrossRef] [PubMed]

22. Vellas, B.; Guigoz, Y.; Garry, P.J.; Nourhashemi, F.; Bennahum, D.; Lauque, S.; Albarede, J.-L. The mini nutritional assessment (MNA) and its use in grading the nutritional state of elderly patients. Nutrition 1999, 15, 116-122. [CrossRef]

23. Hayes, A.F.; Preacher, K.J. Statistical mediation analysis with a multicategorical independent variable. Br. J. Math. Stat. Psychol. 2014, 67, 451-470. [CrossRef]

24. Yoshimura, N.; Muraki, S.; Oka, H.; Iidaka, T.; Kodama, R.; Kawaguchi, H.; Nakamura, K.; Tanaka, S.; Akune, T. Is osteoporosis a predictor for future sarcopenia or vice versa? Four-year observations between the second and third ROAD study surveys. Osteoporos. Int. 2017, 28, 189-199. [CrossRef] [PubMed]

25. Okamura, H.; Ishikawa, K.; Kudo, Y.; Matsuoka, A.; Maruyama, H.; Emori, H.; Yamamura, R.; Hayakawa, C.; Tani, S.; Tsuchiya, K.; et al. Risk factors predicting osteosarcopenia in postmenopausal women with osteoporosis: A retrospective study. PLoS ONE 2020, 15, e0237454. [CrossRef] [PubMed]

26. Nielsen, B.R.; Abdulla, J.; Andersen, H.E.; Schwarz, P.; Suetta, C. Sarcopenia and osteoporosis in older people: A systematic review and meta-analysis. Eur. Geriatr. Med. 2018, 9, 419-434. [CrossRef]

27. Schaap, L.; Pluijm, S.M.F.; Deeg, D.J.H.; Harris, T.B.; Kritchevsky, S.B.; Newman, A.B.; Colbert, L.H.; Pahor, M.; Rubin, S.M.; Tylavsky, F.A.; et al. Higher Inflammatory Marker Levels in Older Persons: Associations with 5-Year Change in Muscle Mass and Muscle Strength. J. Gerontol. Ser. A Boil. Sci. Med. Sci. 2009, 64, 1183-1189. [CrossRef] 
28. Ginaldi, L.; Di Benedetto, M.C.; De Martinis, M. Osteoporosis, inflammation and ageing. Immun. Ageing 2005, 2, 14. [CrossRef]

29. Lim, J.P.; Chong, M.S.; Tay, L.; Yang, Y.X.; Leung, B.P.; Yeo, A.; Yew, S.; Tan, C.H.; Lim, W.S. Inter-muscular adipose tissue is associated with adipose tissue inflammation and poorer functional performance in central adiposity. Arch. Gerontol. Geriatr. 2019, 81, 1-7. [CrossRef]

30. Kalinkovich, A.; Livshits, G. Sarcopenic obesity or obese sarcopenia: A cross talk between age-associated adipose tissue and skeletal muscle inflammation as a main mechanism of the pathogenesis. Ageing Res. Rev. 2017, 35, 200-221. [CrossRef]

31. Barbour, K.E.; Lui, L.-Y.; Ensrud, K.E.; Hillier, T.A.; Leblanc, E.S.; Ing, S.W.; Hochberg, M.C.; Cauley, J.A.; Study of Osteoporotic Fractures (SOF) Research Group. Inflammatory markers and risk of hip fracture in older white women: The study of osteoporotic fractures. J. Bone Miner. Res. 2014, 29, 2057-2064. [CrossRef] [PubMed]

32. Perrini, S.; Laviola, L.; Carreira, M.C.; Cignarelli, A.; Natalicchio, A.; Giorgino, F. The GH/IGF1 axis and signaling pathways in the muscle and bone: Mechanisms underlying age-related skeletal muscle wasting and osteoporosis. J. Endocrinol. 2010, 205, 201-210. [CrossRef]

33. Doi, T.; Makizako, H.; Tsutsumimoto, K.; Hotta, R.; Nakakubo, S.; Makino, K.; Suzuki, T.; Shimada, H. Association between Insulin-Like Growth Factor-1 and Frailty among Older Adults. J. Nutr. Health Aging 2018, 22, 68-72. [CrossRef] [PubMed]

34. Reiss, J.; Iglseder, B.; Alzner, R.; Mayr-Pirker, B.; Pirich, C.; Kässmann, H.; Kreutzer, M.; Dovjak, P.; Reiter, R. Sarcopenia and osteoporosis are interrelated in geriatric inpatients. Z. Gerontol. Geriatr. 2019, 52, 688-693. [CrossRef]

35. Huo, Y.R.; Suriyaarachchi, P.; Gomez, F.; Curcio, C.-L.; Boersma, D.; Gunawardene, P.; Demontiero, O.; Duque, G. Comprehensive nutritional status in sarco-osteoporotic older fallers. J. Nutr. Health Aging 2014, 19, 474-480. [CrossRef]

36. O'Connell, M.L.; Coppinger, T.; McCarthy, A.L. The role of nutrition and physical activity in frailty: A review. Clin. Nutr. ESPEN 2020, 35, 1-11. [CrossRef]

37. Pek, K.; Chew, J.; Lim, J.P.; Yew, S.; Tan, C.N.; Yeo, A.; Ding, Y.Y.; Lim, W.S. Social Frailty Is Independently Associated with Mood, Nutrition, Physical Performance, and Physical Activity: Insights from a Theory-Guided Approach. Int. J. Environ. Res. Public Health 2020, 17, 4239. [CrossRef]

38. Lau, S.; Pek, K.; Chew, J.; Lim, J.P.; Ismail, N.H.; Ding, Y.Y.; Cesari, M.; Lim, W.S. The Simplified Nutritional Appetite Questionnaire (SNAQ) as a Screening Tool for Risk of Malnutrition: Optimal Cutoff, Factor Structure, and Validation in Healthy Community-Dwelling Older Adults. Nutrients 2020, 12, 2885. [CrossRef]

39. Lim, W.; Liang, C.; Assantachai, P.; Auyeung, T.W.; Kang, L.; Lee, W.-J.; Lim, J.; Sugimoto, K.; Akishita, M.; Chia, S.; et al. COVID-19 and older people in Asia: Asian Working Group for Sarcopenia calls to actions. Geriatr. Gerontol. Int. 2020, 20, 547-558. [CrossRef]

(C) 2020 by the authors. Licensee MDPI, Basel, Switzerland. This article is an open access article distributed under the terms and conditions of the Creative Commons Attribution (CC BY) license (http://creativecommons.org/licenses/by/4.0/). 